\title{
Die Unterschiede zwischen Vor-Rcküfußtechnick für Tischtennisspieler
}

\author{
Walaa eldin A. Hazzaah
}

Fakultät für Leibeserziehung, AlHaram, Universität Helwan, Ägypten

\section{Zusammenfassung}

Hintergrund: Sowohl die Fußaufsatztechnik als auch die lokale Muskelermüdung können zur Änderung der plantaren Druckverteilung unter den Fußbereichen führen, was die Verletzungsrate im Tischtennissport erhöhen kann.

Methoden: An der Studie nahmen 48 Tischtennisspieler, mit unterschiedlicher Fußaufsatztechnik (Vorfuß versus Rückfuß) teil. Der Testablauf umfasste insgesamt drei Termine. Eine gesonderte Sitzung, um die Studienteilnehmer mit den Tests sowie den Messgeräten vertraut zu machen. Der Maximalkrafttest umfasste zwei Sätze mit einer maximalen Kontraktion. Das isokinetische Ermüdungsprotokoll setzte sich aus zehn Sätze a sechs Wiederholungen konzentrischer Kontraktionen der Plantar- und Dorsalflexoren mit einer Winkelgeschwindigkeit von $60 \%$ zusammen. Zur Überprüfung der Unterschiede wurde eine zweifaktorielle Varianzanalyse mit Messwiederholung berechnet.

Ergebnisse: Die Rückfuß Tischtennisspieler realisierten im Durchschnitt links höhere und rechts niedrigere Werte als die Vorfuß Tischtennisspieler. Der Symmetrieindex der Druckverteilung unter der Ferse wies eine höhere Asymmetrie bei den Vorfuß Tischtennisspieler gegenüber Rückfuß Tischtennisspieler auf. Rückfuß Tischtennisspieler realisieren höhere plantare Druckmaxima unter der Ferse und geringere Druckmaxima unter dem Vorfuß im Vergleich zu Vorfuß Tischtennisspieler.

Schlussfolgerung: Die plantare Druckverteilung unterscheidet sich unter dem Fuß in Abhängigkeit von der Fußaufsatztechnik und der lokalen Muskelermüdung. Eine Verringerung des plantaren Druckmaximums unter der rechten Ferse gab es bei den Rückfuß Tischtennisspieler und eine Verringerung des plantaren Druckmaximums unter dem Vorfuß bei beiden Tischtennis Gruppen unter dem linken und dem rechten Bein aufgrund der lokalen Ermüdung.

\section{Schlüsselworter: Vorfußaufsatz, Rückfußaufsatz, Asymmetrie, Krafttest}

\section{Einleitung}

Die Menschen werden geboren, mit der Fähigkeit zu Laufen. Bramble et al. (2004) gehen davon aus, dass unsere Körperstruktur beeinflusst wurde, durch die Tatsache, dass die Menschen das Laufen zum Überleben brauchen (Bramble et al., 2004). Heutzutage kann Laufen als eine der wichtigsten Freizeitaktivitäten in Betracht gezogen werden (De Wit et al., 2000). Laufen ist eine einfache und kostengünstige Lösung für viele, die Gewicht verlieren möchten, Stress abbauen oder ihre kardiovaskuläre Fitness verbessern möchten. Die Beliebtheit am Tischennis spielen hat sich in den vergangenen Jahren erhöht und mit Anstieg der Popularität sind auch die laufassoziierten Beschwerden angestiegen (Daoud et al., 2012).

Trotz der Tatsache, dass es in den letzten Jahrzehnten viele wissenschaftliche Untersuchungen gab, um die Verletzungsrate im Laufsport zu vermindern, ist diese auf dem gleichen Niveau geblieben (Richards et al., 2009). Tischtennis spielen hat viele positive Auswirkungen auf den Körper ,erhöht aber auch die Gefahr von Verletzungen der unteren Extremität(Landreneau et al., 2014) Mit dem Zuwachs an Tischtennis spielen wurde eine Erhöhung der klassischen belastungsabhängigen Beschwerden des Stütz- und Bewegungsapparates erfasst(Dickhuth et al., 2001) und die gesamte Beschwerdeinzidenz ist weiter angestiegen(Novackeck, 1998).Gegenwärtig geht man von einer Verletzungsquote von ca. $30 \%$ bei Läufern aus.(Dickkuth et al.,2001).Dabei betreffen über 90 Prozent der laufassoziierten Beschwerden die untere Extremität, wovon ca. ein Drittel jeweils auf das Knie, den Unterschenkel und den Fuß entfallen (Fredericson et al., 2007; Hreljac, 2005). 
Die Fußaufsatztechnik sollte differenziert betrachtet werden, um in der Praxis individuelle Trainingsansätze erstellen zu können und Verletzung im Vorfeld zu vermeiden (Cavanagh et al., 1980; Willems et al., 2012; Milner et al., 2006).

Die Beziehung zwischen Fußaufsatz und Verletzung ist nicht eindeutig geklärt (James et al., 1978; Bennell et al., 2005).Mehrere Studien haben gezeigt, dass ein Zusammenhang zwischen unterschiedlichem Fußaufsatz und Verletzungen beim Tischtennisspielen besteht (Williams et al., 2000; Milner et al., 2006).Aufgrund der unterschiedlichen Untersuchungsergebnisse zur Fußaufsatztechnik werden Empfehlungen zur Bewegungen beim Tischtennis kontrovers diskutiert.

Die Unterschiede der Fußstellung bei der Landung klassifizieren die verschiedenen Bewegungsstile. Üblicherweise wird der Fußaufsatz definiert über den Teil des Fußes, der beim Laufen den ersten Kontakt mit dem Boden hat und normalerweise wird dabei in drei Kategorien unterschieden: 1. Rückfußaufsatz, wenn der Läufer zuerst auf der Ferse landet, 2. Mittelfußaufsatz, wenn der Läufer gleichzeitig auf der Ferse und dem Fußballen landet ; 3. Vorfußaufsatz, wenn die erste Landung auf dem Fußballen stattfindet(Fellin et al., 2010; Davis et al., 2010; Lieberman et al., 2010: Cavanagh et al., 1980;Bobbert et al., 1992; Altman et al., 2012).Trotz dieser Unterteilung in drei Fußaufsatztechniken, konzentrieren sich die meisten Studien auf Untersuchungen zwischen Rück und Vorfußspieler. (Hasegawa et al., 2007; Kerr et al.,1983; Ahn et al., 2014 Landreneau et al.,2014; Liebl et al., 2014).Neben Fußaufsatztechnik gibt es viele Faktoren, die Einfluss auf die Erhöhung der Verletzungsgefahr beim Laufen haben, unter anderem die muskuläre Ermüdung. (Cavanagh et al., 1980; Burr, 1997; García-Pérez et al., 2013;Kellis et al., 2009; Baker et al.,1972; Dickinson et al.,1985; Grimston et al., 1993; Nyland et al., 1994 ) Es ist davon auszugehen, dass Ermüdung die Fußaufsatztechnik beeinflusst, obwohl der Einfluss der Ermüdung auf die Fußaufsatztechnik noch nicht ausreichend geklärt wurde. (De Almeida et al.,2015).Trotz zahlreicher Studien liegen keine eindeutigen Befunde vor, die eine relevante Aussage machen können, wie der Fußaufsatz sich verändert nach Ermüdung. Um die Verletzung vorzubeugen und Trainingspläne zu erstellen, muss herausgefunden werden, wie sich die Fußaufsatztechnik durch Ermüdung verändert. Dabei kann jedoch nicht differenziert werden, welche kinematischen oder kinetischen Veränderungen unmittelbares Ergebnis der lokalen Muskelermüdung darstellen. Für eine gezielte Analyse bieten sich standardisierte lokale Ermüdungsprotokolle der Muskulatur des Knie- und/oder Sprunggelenks vor dem Laufen an. Da die Dorsal- und Plantarflexoren des Fußes zwischen 50 - 85\% des Laufzyklus aktiv sind (Reber et al. 1993, Kellis et al.2009), können diese folglich stark ermüden.

Studien zur Nutzung der Fußaufsatztechnik liefern widersprüchliche Befunde. Mehrere Studien stellten fest, dass der überwiegende Teil an Spieleren Rückfuß sind, während andere, den Vorfuß als natürlichen Spielart bezeichnen und somit das Verletzungsrisiko geringer ist.

Liebermann et al. (2010)\& Wiliams et al.(2000) haben herausgefunden, dass die Landung-und die Bodenreaktionskräfte bei Vorfuß Tischtennisspieler niedriger sind. Die Autoren fanden heraus,dass die Landung bei Vorfuß Tischtennisspieler 7 mal niedriger ist als bei Rückfuß Tischtennisspieler (Liebermann et al. ,2010; Wiliams et al.,2000). In einer Studie mit 19 Läufern fanden Kleindienst et al. (2007) aufgrund der kinematischen Daten kein geringeres Verletzungsrisiko der Vorfuß gegenüber der Rückfuß, wobei es jedoch einen Zusammenhang zwischen Fußaufsatztechnik und Verletzungsbereich gibt. Arendse et al. (2004) zeigten in einer Studie ein erhöhtes Verletzungsrisiko für Vorfuß Tischtennisspieler. Allerdings nicht alle Studien konnten einen Zusammenhang zwischen Fußaufsatztechnik und Höhe des Verletzungsrisiko feststellen. (Bredeweg et al. ,2011;Nigg et al. ,1995). Vorfuß Tischtennisspieler zeichnen sich aus durch eine gekennzeichnete abgeschwächte oder nicht vorhandene erste passive Spitze aber eine erhöhte aktive Spitze (Laughton et al., 2003; Liebermann et al.,2010). Laughton et al. (2013) haben keine Unterschiede zwischen Vor-und Rückfuß Tischtennisspieler bei der Landung und der Bodenreaktionskraft gefunden. Ziel der Studie war die Untersuchung des Einflusses der Fußaufsatztechnik und der lokalen Muskelermüdung der Plantar- und Dorsalflexoren auf die plantare Druckverteilung beim Laufen für Tischtennisspieler. Es wurde erwartet, dass sich die plantare Druckverteilung unter dem Fuß in Abhängigkeit von der Fußaufsatztechnik und der lokalen Muskelermüdung unterscheiden. Rückfuß Tischtennisspieler realisieren höhere plantare Druckmaxima unter der Ferse und geringere Druckmaxima unter dem 
Vorfuß im Vergleich zu Vorfuß Tischtennisspieler. Infolge der lokalen Ermüdung der Plantar- und Dorsalflexoren reduzieren sich die plantaren Druckmaxima unter dem Fuß bei beiden Fußaufsatztechniken.

\section{Material und Methoden}

\section{Studiendesign und Stichprobe}

Die Studie war als Querschnittsvergleich von zwei freiwilligen Tischtennisspieler vergleichbaren Alters und Körpermasse, aber unterschiedlicher Fußaufsatztechnik (Vorfuß Tischtennisspieler versus Rückfuß Tischtennisspieler) angelegt. Im Durchschnitt absolvierten die Tischtennisspieler wöchentlich zwei bis drei Laufeinheiten. Alle Tischtennisspieler waren zum Messzeitpunkt gesund, ohne aktuelle orthopädische Befunde.Vor Studienbeginn wurde ein Ethikvotum bei der Ärztekammer Hamburg eingeholt.

Tab. 1 Übersicht über die Untersuchungsgruppen, $\mathrm{N}=48$

\begin{tabular}{|c|c|c|c|c|}
\hline Gruppe & Anzahl & Alter [J] & Körpermasse [kg] & Körperhöhe [cm] \\
\hline Vorfuß & 24 & $25,9 \pm 3,8$ & $82,0 \pm 8,9$ & $183,5 \pm 7,3$ \\
\hline Rückfuß & 24 & $25,9 \pm 3,8$ & $81,0 \pm 8,7$ & $182,5 \pm 6,7$ \\
\hline Gesamt & 48 & $25,9 \pm 3,8$ & $81,5 \pm 8,8$ & $183,0 \pm 7$, \\
\hline
\end{tabular}

\section{Untersuchungsablauf}

Der Testablauf umfasste insgesamt drei Termine. Eine gesonderte Sitzung, um die Studienteilnehmer mit den Tests sowie den Messgeräten vertraut gemacht, sowie zwei Tests, die im Abstand von drei bis sieben Tagen absolviert wurden. Dargestellt werden die Ergebnisdaten von Test 2, weil keine signifikanten Unterschiede der Ergebnisse von Test 1 und 2 vorlagen. Am Testtag führten die Tischtennisspieler ein 10-minütiges Laufen auf dem Laufband mit einer Geschwindigkeit von $9 \mathrm{~km} / \mathrm{h}$ als Erwärmung und zur Gewöhnung an das Laufband durch. Danach absolvierten die Teilnehmer den isometrischen Maximalkrafttest in randomisierter Reihenfolge für das linke und rechte Bein. Danach folgten das isokinetische Ermüdungsprotokoll für ein Bein und unmittelbar danach der Laufbandtest. Dem schloss sich wiederum das Ermüdungsprotokoll des anderen Beines gefolgt vom Laufbandtest an.

\section{Testbeschreibung}

Die Eingewöhnung umfasste das Üben des Laufens auf dem Laufband von 15 Minuten sowie die probeweise Durchführung der Krafttests. Dabei wurden die Fußaufsatztechnik erfasst und die Positionierungsdaten für den Krafttest registriert und die Einstellungen im Kraftmessgerät gespeichert. Zur Gewöhnung an das Testgerät und zum Aufwärmen der Zielmuskulatur wurden dem isokinetischen Maximalkrafttest eine submaximal Kontraktionen im Gerät vorgeschaltet, um die Probanden an den Test zu gewöhnen und die Zielmuskulatur aufzuwärmen. Zur Positionierung wurden die Herstellerangaben verwendet. Der Proband befand sich in sitzender Position, der Oberschenkel war fixiert und jeweils ein Winkel von $90^{\circ}$ in Knie- und Sprunggelenk eingestellt. Die Drehachse von oberem Sprunggelenk (dickste Stelle des Malleolus lateralis zum medialen Malleolus) und vom Dynamometer wurden per Laser justiert. Der Fuß war mit einem Gurt auf der Platte befestigt. Die Neigung der Rückenlehne betrug $70^{\circ}$. Der Maximalkrafttest umfasste zwei Sätze mit einer maximalen Kontraktion. Die Kraft sollte dabei rampenförmig bis zum Maximum nach 2- $3 \mathrm{~s}$ aufgebaut werden. Die Satzpause betrug drei Minuten. Das isokinetische Ermüdungsprotokoll setzte sich aus zehn Sätzen a sechs Wiederholungen konzentrischer Kontraktionen der Plantar- und Dorsalflexoren mit einer Winkelgeschwindigkeit von 60 \% zusammen. Die Satzpause betrug $10 \mathrm{~s}$. Das Bewegungsausmaß war auf maximal $55^{\circ}$ für die Plantarflexion und $25^{\circ}$ für die Dorsalflexion eingestellt. Begonnen wurde mit der Plantarflexion.

\section{Messgeräte}

Die Tests zur Maximalkraft der Plantar- und Dorsalflexion im Sprunggelenk erfolgten mit dem Isomed 2000-Dynamometer (D\&R Ferst/GmbH, Hemau, Germany) unter Verwendung des vom Hersteller entwickelten Adapters. Die Genauigkeit der Messung betrug beim Drehmoment 0,25\% bei 
einer Messfrequenz von $200 \mathrm{~Hz}$. Das Gerät erfüllte medizintechnische Sicherheitsstandards. Die Erhebung und Bereitstellung der Testergebnisse erfolgte mit der Computer Software des Herstellers (IsoMed analyze 2008). Aus dem isometrischen Maximalkrafttest wurde das jeweils höhere maximale Drehmoment eines Satzes als Testwert (Mx) erhoben. Für den isokinetischen Test wurde der Mittelwert der Maxima des Drehmoments aus allen 60 Kontraktionen (M60) ermittelt. Im TestRetest-Vergleich konnte für das Ermüdungsprotokoll der Plantar- und Dorsalflexoren (M60) eine hohe relative Reproduzierbarkeit mit ICC(3.1)-Werten von im Durchschnitt größer als 0,9 festgestellt werden. Der errechnete Ermüdungsindex (Mx/M60) zeigte für die Plantarflexoren eine hohe $(0,92)$ und für die Dorsalflexoren eine moderate relative Reproduzierbarkeit $(0,88)$ (Mattes et al., 2015).

\section{Mathematisch-statistische Auswertung}

Der Ermüdungsindex wurde als Quotient des Mittelwertes der Drehmonentenmaxima aus dem Kraftausdauertest geteilt durch das maximale Drehmoment aus dem isometrischen Maximalkrafttest nach der Formel: Ermüdungsindex= Mx/M60 für die Plantar- und Dorsalflexion gebildet. Ein kleiner Koeffizient zeigt dabei eine starke lokale Muskelermüdung an. Um den Grad der Asymmetrie zwischen linkem und rechtem Bein zu bestimmen, wurde der Symmetrieindex (SI) nach der Formel: $\mathrm{SI}=\frac{\mathrm{I}(\mathrm{xl}-\mathrm{xr}) \mathrm{I}}{0,5(\mathrm{xl}+\mathrm{xr})} 100$

berechnet (Zifchock et al. 2008). Dabei wurden für xl und xr die jeweiligen Messdaten der Plantaroder Dorsalflexion des linken und rechten Beines eingesetzt.

Die statistische Auswertung umfasste die Beschreibung mit arithmetischem Mittelwert und Standardabweichung. Mittels dem Kolmogorov-Smirnov und dem Levene-Test wurden die Daten auf Normalverteilung und Varianzhomogenität geprüft. Zur Überprüfung der Unterschiede wurde eine zweifaktorielle Varianzanalyse mit Messwiederholung nach dem allgemeinen linearen Modell und dem Innersubjektfaktoren Bein (links und rechts) und Test (Baseline, Ermüdung links und Ermüdung rechts) sowie dem Zwischensubjektfaktor Fußaufsatz (Vorfuß Tischtennisspieler versus Rückfuß Tischtennisspieler) berechnet werden. Für den Symmetrieindex kam eine einfaktorielle Varianzanalyse mit Messwiederholung mit dem Innersubjektfaktor Test und dem Zwischensubjektfaktor Fußaufsatz zur Anwendung. Zur Prüfung der paarweisen Mittelwertunterschiede wurde der LSD (Least Significant Difference) herangezogen. Das partielle Eta-Quadrat ( $\eta \mathrm{p} 2$ ) diente als Maß der Effektstärke (kleiner Effekt $\eta p 2 \geq 0,08$, mittlerer Effekt $\eta p 2 \geq 0,20$, großer Effekt $\eta p 2 \geq 0,32$ (Cohen, 1988 und 1992). Für sämtliche statistischen Tests war ein Signifikanzniveau mit $\mathrm{p} \leq 0,05$ festgelegt. Die statistischen Berechnungen erfolgten mittels IBM SPSS 20.0 (Chicago, IL, USA).

\section{Ergebnisse \\ Krafttest}

Die Kraftwerte der beiden Läufergruppen differierten bei den Plantarflexoren im Kraftausdauertest und beim Ermüdungsindex mit im Durchschnitt höheren Werten der Rückfuß Tischtennisspieler (Tab. 2). Bei den Dorsalflexoren zeigten die Rückfuß Tischtennisspieler höhere isometrische Maximalkraftwerte und aufgrund vergleichbarer Kraftausdauerergebnisse ein im Durchschnitt geringeren Ermüdungsindex als die Vorfuß Tischtennisspieler.

Ein Haupteffekt des Beines bestand bei den Plantarflexoren beim maximalen isometrischen Drehmoment mit höheren Werten des linken Beins $(\mathrm{p}=, 023)$ und damit auch beim Ermüdungsindex mit geringen Werten des linken Beins $(\mathrm{p}=, 021)$. Bei den Plantarflexoren bestand zudem eine Wechselwirkung Bein*Fußaufsatz beim maximalen isometrischen Drehmonent $(p=, 015)$. Die Rückfuß Tischtennisspieler realisierten im Durchschnitt links höhere und rechts niedrigere Werte als die Vorfuß Tischtennisspieler. Der Links-/ Rechtsvergleich bei den Dorsalflexoren differierte beim maximalen isokinetischen Drehmoment im Ausdauertest $(\mathrm{p}=, 019)$ sowie beim Ermüdungsindex $(\mathrm{p}=, 010)$ mit höheren Werten des linken Beines. Dabei ist ebenfalls die Wechselwirkung Bein*Fußaufsatz $(\mathrm{p}=, 000)$ zu beachten. Im Unterschied zu den Rückfuß Tischtennisspieler realisierten die Vorfuß Tischtennisspieler im Ausdauertest im Mittel etwas höhere Werte mit dem rechten Bein im Vergleich zum linken Bein (Tab. 2). 
Beim Asymmetrieindex wurden bei den Plantorflexoren beim maximalen isometrischen Drehmoment und bei den Dorsalflexoren beim isokinetischen Kraftausdauertest höhere Links-Rechtsunterschiede bei den Vorfuß Tischtennisspieler im Vergleich zu den Rückfuß Tischtennisspieler gefunden (Tab. 2).

Tab. 2 Ergebnisse des Krafttests, Mittelwert \pm Standardabweichung sowie Daten der Varianzanalyse, maximales isometrisches Drehmoment (Mx), Mittelwert der maximalen Drehmomente aus dem Kraftausdauertest (M60), Ermüdungsindex (M60/Mx), Symmetrieindex (SI), Prüfwerte des Zwischensubjekteffekts Fußaufsatz (F, p und $\eta p 2), \mathrm{N}=48$

\begin{tabular}{|c|c|c|c|c|c|c|c|c|}
\hline \multirow{2}{*}{ Muskel } & \multirow{2}{*}{ Kennwert } & \multirow{2}{*}{ Bein } & \multicolumn{3}{|c|}{ Fußaufsatz } & \multicolumn{3}{|c|}{ Zwischensubjekteffekt Fußaufsatz } \\
\hline & & & Vorfuß & Rückfuß & Gesamt & $\mathrm{F}$ & $\mathrm{P}$ & Пр2 \\
\hline \multirow{9}{*}{$\begin{array}{l}\text { Plantar- } \\
\text { flexoren }\end{array}$} & \multirow{2}{*}{$\mathrm{Mx} 1,4[\mathrm{Nm}]$} & links & $145 \pm 21$ & $165 \pm 27$ & $155 \pm 24$ & \multirow{2}{*}{,659 } & \multirow{2}{*}{,391 } & \multirow{2}{*}{, 014} \\
\hline & & rechts & $144 \pm 30$ & $162 \pm 32$ & $153 \pm 31$ & & & \\
\hline & \multirow{2}{*}{$\mathrm{M} 60[\mathrm{Nm}]$} & links & $42 \pm 14$ & $77 \pm 28$ & $60 \pm 21$ & \multirow{2}{*}{62,0} & \multirow{2}{*}{, 000} & \multirow{2}{*}{,495 } \\
\hline & & rechts & $49 \pm 16$ & $83 \pm 28$ & $66 \pm 22$ & & & \\
\hline & \multirow{2}{*}{ M60/Mx1 } & links & $0,40 \pm 0,11$ & $0,57 \pm 0,14$ & $0,48 \pm 0,13$ & \multirow{2}{*}{60,6} & \multirow{2}{*}{, 000} & \multirow{2}{*}{,498 } \\
\hline & & rechts & $0,37 \pm 0,13$ & $0,59 \pm 0,17$ & $0,48 \pm 0,15$ & & & \\
\hline & SIMx [\%] & \multirow{3}{*}{$\begin{array}{l}\text { links und } \\
\text { rechts }\end{array}$} & $33,6 \pm 12,2$ & $21 \pm 18,8$ & $27,3 \pm 16,9$ & 8,3 & 0,01 & \\
\hline & SIM60 [\%] & & $40,1 \pm 39,7$ & $21,9 \pm 14,4$ & $31 \pm 30,9$ & 4,8 & 0,03 & \\
\hline & SIM60/Mx [\%] & & $27,9 \pm 36,2$ & $20,8 \pm 16,2$ & $24,3 \pm 28$ & 0,8 & 0,37 & \\
\hline \multirow{9}{*}{$\begin{array}{l}\text { Dorsal- } \\
\text { flexoren }\end{array}$} & \multirow{2}{*}{$\mathrm{Mx}[\mathrm{Nm}]$} & links & $24,2 \pm 10$ & $34,8 \pm 10$ & $29,5 \pm 10$ & \multirow{2}{*}{8,5} & \multirow{2}{*}{, 005} & \multirow{2}{*}{, 146} \\
\hline & & rechts & $29 \pm 8,3$ & $36,3 \pm 13,2$ & $33,1 \pm 11$ & & & \\
\hline & \multirow{2}{*}{$\mathrm{M} 601,4[\mathrm{Nm}]$} & links & $15,6 \pm 3,7$ & $17,2 \pm 4,3$ & $16,4 \pm 4$ & \multirow{2}{*}{,01 } & \multirow{2}{*}{,920 } & \multirow{2}{*}{, 000} \\
\hline & & rechts & $12,6 \pm 3,1$ & $13,5 \pm 4,3$ & $12,9 \pm 3,3$ & & & \\
\hline & \multirow{2}{*}{ M60/Mx1 } & links & $0,53 \pm 0,17$ & $0,51 \pm 0,25$ & $0,57 \pm 0,21$ & \multirow{2}{*}{11,0} & \multirow{2}{*}{,002 } & \multirow{2}{*}{, 180} \\
\hline & & rechts & $0,44 \pm 0,13$ & $0,40 \pm 0,17$ & $0,42 \pm 0,15$ & & & \\
\hline & SIMx [\%] & \multirow{3}{*}{$\begin{array}{l}\text { links und } \\
\text { rechts }\end{array}$} & $45,6 \pm 21,1$ & $25,2 \pm 20,1$ & $35,4 \pm 22,9$ & 12,8 & 0,00 & \\
\hline & SIM60 [\%] & & $39,3 \pm 16,8$ & $32,3 \pm 20,4$ & $35,8 \pm 18,9$ & 1,9 & 0,18 & \\
\hline & SIM60/Mx [\%] & & $21 \pm 14,9$ & $28,3 \pm 19,7$ & $24,7 \pm 17,7$ & 2,3 & 0,14 & \\
\hline
\end{tabular}

1 signifikanter Haupteffekt des Beines; 4 signifikante Wechselwirkung Bein*Fußaufsatz

\section{Laufbandtest}

Den Lauftest absolvierten beide Stichproben mit der gleichen Laufgeschwindigkeit sowie vergleichbarer Doppelschrittlänge,schrittfrequenz und Schrittlänge. Die beiden Fußaufsatztechniken führten zu markanten Unterschieden in der plantaren Druckverteilung

Bei den Rückfuß Tischtennisspieler traten höhere mittlere plantare Druckmaxima unter dem Rückfuß bei zugleich geringen maximalen Druckwerten unter dem Mittel- und Vorfuß im Vergleich zu den Vorfuß Tischtennisspieler auf. Der Symmetrieindex der Druckverteilung unter der Ferse wies eine höhere Asymmetrie bei den Vorfuß Tischtennisspieler gegenüber Rückfuß Tischtennisspieler auf (Tab. 3).

Die lokale Muskelermüdung beeinflusste die plantaren Druckmaxima unter der rechten Ferse $(p=0,02)$, wobei auch eine Wechselwirkung Ermüdung*Fußaufsatz $(p=0,00)$ bestand. Die Rückfuß Tischtennisspieler verringerten nach dem Ermüdungsprotokoll in allen drei Geschwindigkeiten die plantaren Druckmaxima unter der rechten Ferse. Bei den Vorfuß Tischtennisspieler waren dagegen keine Veränderungen der Druckmaxima unter der Ferse festzustellen. Beide Läufergruppen 
reduzierten nach dem Ermüdungsprotokoll die plantaren Druckmaxima unter dem linken und rechten Vorfuß in allen drei Geschwindigkeiten. Das erste Kraftmaximum reduzierte sich bei beiden Läufergruppen nach dem Ermüdungsprotokoll im Vergleich zur Baseline in allen drei Laufgeschwindigkeiten (Tab. 3).

Der Einfluss der Laufgeschwindigkeit zeigte sich bei allen untersuchten kinematischen und dynamischen Kennwerten, wobei mit der Laufgeschwindigkeit in beiden Läufergruppen die Werte systematisch anstiegen (Tab. 3).

Tab. 3 Ergebnisse des Lauftests, Mittelwert \pm Standardabweichung sowie Daten der Varianzanalyse, Baseline (Base), Ermüdung links (Eli) und rechts (Ere), Doppelschrittlänge $(s D)$, Schrittfrequenz (f), Schrittlänge (Sl), Druckmaximum unter der Ferse (pxh), dem Mittelfu $\beta$ (pxm) und dem Vorfu $\beta$ (pxf), Kraftmaximum (Fx) und Symmetrieindex (SI), Prüfwerte des Zwischensubjekteffekts Fußaufsatz (F, p und $\eta p 2), N=48$

\begin{tabular}{|c|c|c|c|c|c|c|c|c|c|c|c|}
\hline \multirow{2}{*}{$\begin{array}{l}\text { Kenn- } \\
\text { wert }\end{array}$} & \multirow{2}{*}{ Test } & \multirow{2}{*}{ Bein } & \multicolumn{2}{|c|}{$\begin{array}{l}\text { Laufgeschwindigke } \\
\text { it } 12 \mathrm{~km} / \mathrm{h}\end{array}$} & \multicolumn{2}{|c|}{$\begin{array}{l}\text { Laufgeschwindigke } \\
\text { it } 14 \mathrm{~km} / \mathrm{h}\end{array}$} & \multicolumn{2}{|c|}{$\begin{array}{l}\text { Laufgeschwindigke } \\
\text { it } 16 \mathrm{~km} / \mathrm{h}\end{array}$} & \multicolumn{3}{|c|}{$\begin{array}{l}\text { Zwischensubjekt- } \\
\text { effekt Fußaufsatz }\end{array}$} \\
\hline & & & Vorfuß & $\begin{array}{c}\text { Rückfu } \\
\beta\end{array}$ & Vorfuß & $\begin{array}{c}\text { Rückfu } \\
\beta\end{array}$ & Vorfuß & $\begin{array}{c}\text { Rückfu } \\
\beta\end{array}$ & $\mathrm{F}$ & $\mathrm{p}$ & $\prod_{2}$ \\
\hline \multirow{3}{*}{$\begin{array}{l}\mathrm{sD} \\
{[\mathrm{cm}]}\end{array}$} & $\begin{array}{c}\text { Base } \\
3\end{array}$ & \multirow{3}{*}{$\begin{array}{l}\text { Links } \\
\text { und } \\
\text { rechts }\end{array}$} & $\begin{array}{c}209 \pm \\
12,4\end{array}$ & $\begin{array}{c}210 \pm \\
13,5\end{array}$ & $\begin{array}{c}235 \pm \\
16,2\end{array}$ & $\begin{array}{c}236 \pm \\
16,5\end{array}$ & $\begin{array}{c}257 \pm \\
18\end{array}$ & $\begin{array}{c}259 \pm \\
19,5\end{array}$ & \multirow{3}{*}{0,5} & \multirow{3}{*}{$\begin{array}{c}0,4 \\
8\end{array}$} & \multirow{3}{*}{$\begin{array}{c}0,0 \\
1\end{array}$} \\
\hline & Eli3 & & $\begin{array}{c}206 \pm \\
14,9 \\
\end{array}$ & $\begin{array}{c}209 \pm \\
14\end{array}$ & $\begin{array}{c}233 \pm \\
18 \\
\end{array}$ & $\begin{array}{c}237 \pm \\
16,1 \\
\end{array}$ & $\begin{array}{l}256 \pm \\
21,2 \\
\end{array}$ & $\begin{array}{c}259 \pm \\
20,2 \\
\end{array}$ & & & \\
\hline & Ere3 & & $\begin{array}{c}208 \pm \\
14,1\end{array}$ & $\begin{array}{c}211 \pm \\
21,6\end{array}$ & $\begin{array}{c}232 \pm \\
19\end{array}$ & $\begin{array}{c}237 \pm \\
16,1\end{array}$ & $\begin{array}{c}254 \pm \\
22,1\end{array}$ & $\begin{array}{l}261 \pm \\
20,5\end{array}$ & & & \\
\hline \multirow{3}{*}{$\mathrm{f}[\mathrm{Hz}]$} & $\begin{array}{c}\text { Base } \\
3\end{array}$ & \multirow{3}{*}{$\begin{array}{l}\text { Links } \\
\text { und } \\
\text { rechts }\end{array}$} & $\begin{array}{c}180 \pm \\
12,7\end{array}$ & $\begin{array}{c}179 \pm \\
13,4\end{array}$ & $\begin{array}{c}189 \pm \\
15,6\end{array}$ & $\begin{array}{c}188 \pm \\
15,8\end{array}$ & $\begin{array}{c}198 \pm \\
16,5\end{array}$ & $\begin{array}{c}197 \pm \\
17,7\end{array}$ & \multirow{3}{*}{0,4} & \multirow{3}{*}{$\begin{array}{c}0,5 \\
5\end{array}$} & \multirow{3}{*}{$\begin{array}{c}0,0 \\
1\end{array}$} \\
\hline & Eli3 & & $\begin{array}{c}183 \pm \\
15,6\end{array}$ & $\begin{array}{c}181 \pm \\
13,4\end{array}$ & $\begin{array}{c}188 \pm \\
15,5\end{array}$ & $\begin{array}{c}188 \pm \\
12,5\end{array}$ & $\begin{array}{c}198 \pm \\
19,2\end{array}$ & $\begin{array}{c}197 \pm \\
14,9\end{array}$ & & & \\
\hline & Ere3 & & $\begin{array}{c}182 \pm \\
14,6\end{array}$ & $\begin{array}{c}181 \pm \\
14,8\end{array}$ & $\begin{array}{c}189 \pm \\
18,8\end{array}$ & $\begin{array}{c}190 \pm \\
17,4\end{array}$ & $\begin{array}{c}199 \pm \\
20,1\end{array}$ & $\begin{array}{c}198 \pm \\
17,4\end{array}$ & & & \\
\hline \multirow{4}{*}{$\begin{array}{l}\mathrm{sL} \\
{[\mathrm{cm}]}\end{array}$} & $\begin{array}{c}\text { Base } \\
3\end{array}$ & \multirow{2}{*}{ Links } & $\begin{array}{c}107 \pm \\
7,3\end{array}$ & $\begin{array}{c}108 \pm \\
6,7\end{array}$ & $\begin{array}{r}120 \\
\pm 7,2 \\
\end{array}$ & $10 \pm 7,3$ & $\begin{array}{c}131 \\
\pm 7,9 \\
\end{array}$ & $\begin{array}{c}132 \\
\pm 10,7\end{array}$ & \multirow{2}{*}{0,4} & \multirow{2}{*}{$\begin{array}{c}0,5 \\
3\end{array}$} & \multirow{2}{*}{$\begin{array}{c}0,0 \\
1\end{array}$} \\
\hline & Eli3 & & $\begin{array}{c}105 \pm \\
6,5\end{array}$ & $\begin{array}{c}107 \pm 8 \\
7\end{array}$ & $\begin{array}{l}119 \pm \\
10,2\end{array}$ & $\begin{array}{l}120 \pm \\
8,7\end{array}$ & $\begin{array}{c}130 \pm \\
11,4\end{array}$ & $\begin{array}{c}131 \pm \\
12,3\end{array}$ & & & \\
\hline & $\begin{array}{c}\text { Base } \\
3 \\
\end{array}$ & \multirow{2}{*}{$\begin{array}{l}\text { Recht } \\
\text { s }\end{array}$} & $\begin{array}{c}106 \pm \\
5,9\end{array}$ & $\begin{array}{c}106 \pm \\
7,1 \\
\end{array}$ & $\begin{array}{c}118 \pm \\
8,7 \\
\end{array}$ & $\begin{array}{c}119 \pm \\
9,4 \\
\end{array}$ & $\begin{array}{c}130 \pm \\
10,3 \\
\end{array}$ & $\begin{array}{c}131 \pm \\
10,7 \\
\end{array}$ & \multirow{2}{*}{0,6} & \multirow{2}{*}{$\begin{array}{c}0,4 \\
5\end{array}$} & \multirow{2}{*}{$\begin{array}{c}0,0 \\
1\end{array}$} \\
\hline & Ere3 & & $\begin{array}{c}105 \pm \\
8,1\end{array}$ & $\begin{array}{c}107 \pm \\
11,7\end{array}$ & $\begin{array}{c}117 \pm \\
10,7\end{array}$ & $\begin{array}{c}120 \pm \\
9,5\end{array}$ & $\begin{array}{c}129 \pm \\
12,6\end{array}$ & $\begin{array}{c}131 \pm \\
11,1 \\
\end{array}$ & & & \\
\hline \multirow{4}{*}{$\begin{array}{c}\mathrm{pxh} \\
{[\mathrm{N} / \mathrm{m} 2} \\
]\end{array}$} & $\begin{array}{c}\text { Base } \\
3\end{array}$ & \multirow{2}{*}{ Links } & $\begin{array}{c}6,5 \pm \\
4,9 \\
\end{array}$ & $\begin{array}{c}53,3 \pm \\
11,5\end{array}$ & $9,8 \pm 5,4$ & $\begin{array}{c}57,7 \pm \\
11,9 \\
\end{array}$ & $\begin{array}{c}8,6 \pm \\
2,7 \\
\end{array}$ & $\begin{array}{c}59,3 \pm \\
14,3 \\
\end{array}$ & \multirow{2}{*}{$\begin{array}{c}321, \\
6\end{array}$} & \multirow{2}{*}{$\begin{array}{c}0,0 \\
0\end{array}$} & \multirow{2}{*}{$\begin{array}{c}0,8 \\
7\end{array}$} \\
\hline & Eli3 & & $\begin{array}{c}6,9 \pm \\
5,2\end{array}$ & $\begin{array}{c}49,8 \pm \\
12,9\end{array}$ & $\begin{array}{c}9,7 \pm \\
6,3 \\
\end{array}$ & $\begin{array}{c}54,7 \pm \\
13,3\end{array}$ & $10,2 \pm 7$ & $\begin{array}{c}55,8 \pm \\
13,9\end{array}$ & & & \\
\hline & $\begin{array}{c}\text { Base } \\
3\end{array}$ & \multirow{2}{*}{$\begin{array}{c}\text { Recht } \\
\mathrm{s}\end{array}$} & $\begin{array}{c}8,2 \pm \\
4,8\end{array}$ & $\begin{array}{c}47,6 \pm \\
11,6\end{array}$ & $\begin{array}{c}8,5 \pm \\
2,9\end{array}$ & $\begin{array}{c}54,3 \pm \\
10,3\end{array}$ & $\begin{array}{c}9,7 \pm \\
6,4\end{array}$ & $\begin{array}{c}58,5 \pm \\
12,1\end{array}$ & & 0,0 & 0,8 \\
\hline & $\begin{array}{c}\text { Ere2, } \\
3 \\
\end{array}$ & & $\begin{array}{c}9,2 \pm \\
3,9 \\
\end{array}$ & $\begin{array}{c}46,7 \pm \\
12,4 \\
\end{array}$ & $8,3 \pm 3,9$ & $\begin{array}{c}53,3 \pm \\
11,7 \\
\end{array}$ & $\begin{array}{c}8,3 \pm \\
3,9 \\
\end{array}$ & $\begin{array}{l}54 \pm \\
12,3 \\
\end{array}$ & 2 & 0 & 6 \\
\hline & $\begin{array}{c}\text { Base } \\
3\end{array}$ & & $\begin{array}{c}33,4 \pm \\
14,6\end{array}$ & $\begin{array}{c}25,7 \pm \\
7,3\end{array}$ & $\begin{array}{c}35,7 \pm \\
11,9\end{array}$ & $\begin{array}{c}25,5 \pm \\
5,7\end{array}$ & $\begin{array}{c}35,7 \pm \\
11,9\end{array}$ & $\begin{array}{c}27,7 \pm \\
8,3\end{array}$ & & 0,0 & 0,1 \\
\hline pxm & Eli3 & Links & $\begin{array}{c}30,7 \pm \\
10,3\end{array}$ & $\begin{array}{r}25,6 \\
\pm 5,9\end{array}$ & $\begin{array}{c}33,7 \pm \\
11,9\end{array}$ & $\begin{array}{c}24,4 \pm \\
4,9\end{array}$ & $\begin{array}{c}33,3 \pm \\
9,7\end{array}$ & $\begin{array}{c}27,4 \pm \\
4,8\end{array}$ & 10,2 & 0 & 7 \\
\hline $\begin{array}{c}{[\mathrm{N} / \mathrm{m} 2} \\
]\end{array}$ & $\begin{array}{c}\text { Base } \\
3\end{array}$ & Recht & $\begin{array}{c}29,9 \pm \\
9,8\end{array}$ & $\begin{array}{c}24,1 \pm \\
5,1\end{array}$ & $\begin{array}{c}32,8 \pm \\
9,8\end{array}$ & $\begin{array}{c}24,8 \pm \\
5,7\end{array}$ & $\begin{array}{c}35,3 \pm \\
9,5\end{array}$ & $\begin{array}{c}26,3 \pm \\
6,3\end{array}$ & & 0,0 & 0,1 \\
\hline & Ere3 & s & $\begin{array}{c}31,1 \pm \\
10,9\end{array}$ & $\begin{array}{c}23,7 \pm \\
6,6\end{array}$ & $\begin{array}{c}29,8 \pm \\
11,1\end{array}$ & $\begin{array}{c}24,7 \pm \\
5,6\end{array}$ & $\begin{array}{c}33,8 \pm \\
11,5\end{array}$ & $\begin{array}{c}26,6 \pm \\
4,8\end{array}$ & 11,5 & 0 & 9 \\
\hline $\begin{array}{c}\mathrm{pxf} \\
{[\mathrm{N} / \mathrm{m} 2}\end{array}$ & $\begin{array}{c}\text { Base } \\
3\end{array}$ & Links & $\begin{array}{c}48,5 \pm \\
8,4\end{array}$ & $\begin{array}{c}44,4 \pm \\
7,5 \\
\end{array}$ & $\begin{array}{c}49,8 \pm \\
9,3\end{array}$ & $\begin{array}{c}45,5 \pm \\
8,7\end{array}$ & $\begin{array}{c}50,8 \pm \\
8,1 \\
\end{array}$ & $\begin{array}{c}47,3 \pm \\
8,6\end{array}$ & 3,8 & 0,0 & 0,0 \\
\hline & Eli2, & & $46,7 \pm$ & $40,3 \pm$ & $48,7 \pm$ & $43,3 \pm$ & $47,8 \pm$ & $46,1 \pm$ & & 6 & \\
\hline
\end{tabular}




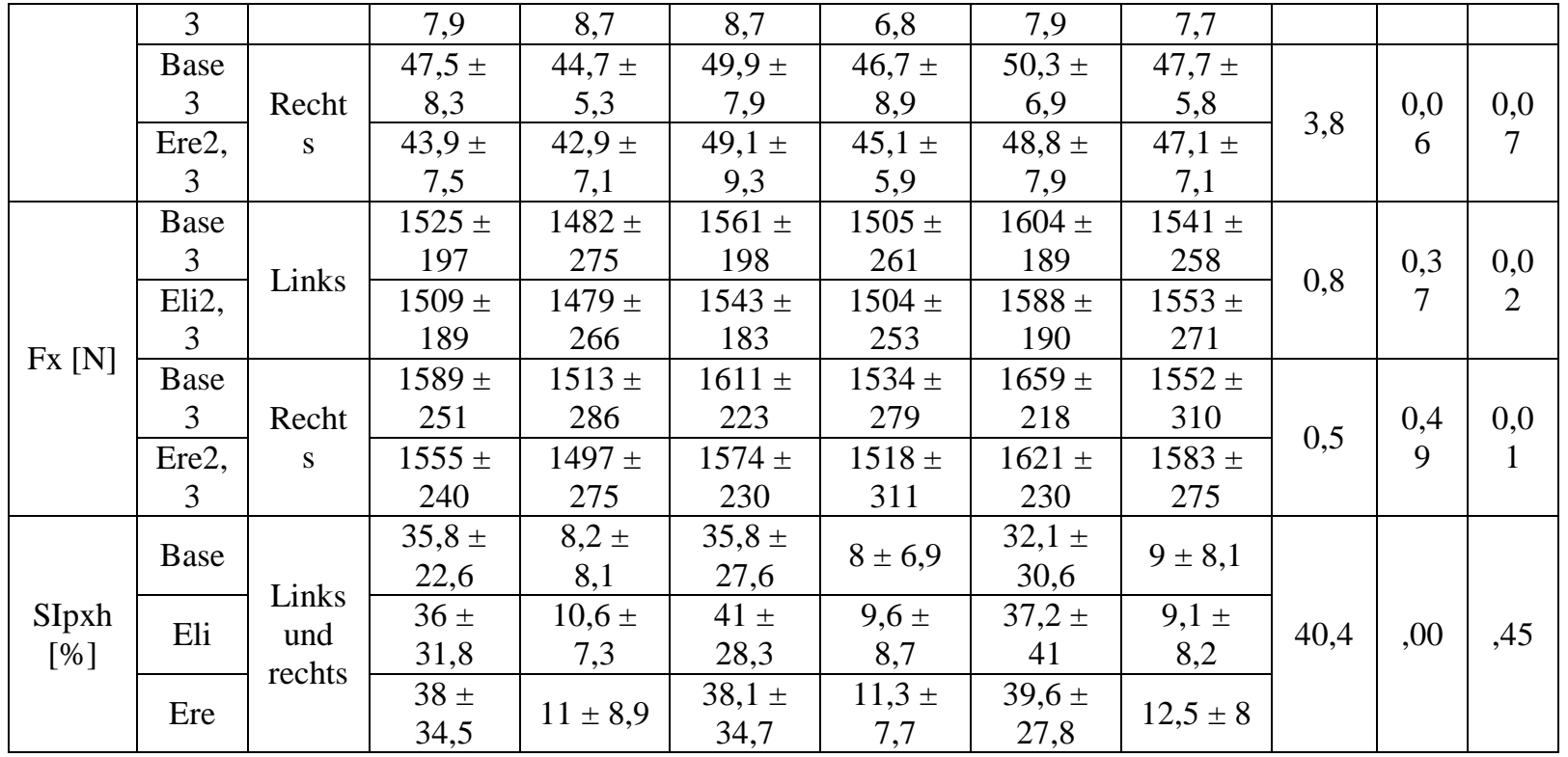

2 signifikanter Haupteffekt der Ermüdung; 3 signifikanter Haupteffekt der Laufgeschwindigkeit

\section{Diskussion}

Primäres Ziel der Studie war die Bestimmung des Einflusses der Fußaufsatztechnik und der lokalen Muskelermüdung der Plantar- und Dorsalflexoren auf die plantare Druckverteilung beim Laufen auf dem Laufband für Tischtennisspieler.

Insgesamt konnten 104 Vergleichsmessungen für beide Tischtennisgruppen vorgenommen werden. In Abhängigkeit von der Fußaufsatztechnik und der lokalen Muskelermüdung wurden unterschiedliche Ergebnisse erzielt. Aufgrund der höheren Kraftwerte der Plantarflexoren vs. Dorsalflexoren kommt es zu einem Anstieg des Verletzungsrisikos (Gerlach et al.,2005; van Mechelen,1992; Baker et al.,1972; Burr et al.,1997; Dickinson et al.,1985; Grimston et al.,1993; Nyland et al.,1994).

Die Werte nach Ermüdung zeigten, dass die Plantarflexoren weniger ermüdet werden, was damit zusammenhängt, dass die ohnehin größere Muskelmasse durch stärkere Beanspruchung der Alltagsbewegungen stärker trainiert sind. Diese muskuläre Dysblance verändert das Abrollverhalten des Fußes, was Auswirkung hat auf die plantare Druckverteilung (Mizrahi et al., 2000).Die Voraktivierung der Plantarflexoren vor der Landung erhöht die Spannung in der Achillessehne und ermöglicht damit eine erhöhte Absorption des Aufpralls bei der Landung (Derrick et al.,1998; Shih et al., 2013; Roberts et al.,2011). Weiterhin führt die Aktivierung der Plantarflexoren dazu, dass die Sehnen im Schaft und im Fuß gedehnt werden, so dass ein verbesserter Speicher von Energie in dieser elastischen Struktur vorhanden ist, damit eine genügende Schockabsorption geleistet werden kann (Perl et al., 2012; Divert et al., 2005). Die Kraftwerte der beiden Läufergruppen differierten bei den Plantarflexoren im Kraftausdauertest und beim Ermüdungsindex mit im Durchschnitt höheren Werten der Rückfuß Tischtennisspieler. Obwohl beim Vorfußlauf die Plantarflexoren stark voraktivieren, um das Körpergewicht bei relativer Fußstellung abzufangen(Landreneau et al.,2014), waren diese bei den Rückfuß Tischtennisspieler unerwartet höher. Beim Rückfußlauf sollte normalerweise die gesamte Aktivität der Plantarflexoren geringer sein(Landreneau et al., 2014).

Die Werte im Kraftausdauertest und der Ermüdungsindex der Plantar-und Dorsalflexoren lagen jedoch im Durschnitt höher bei den Rückfuß Tischtennisspieler als bei den Vorfuß Tischtennisspieler. Wie mehrere Studien zeigten eignet sich der Rückfußlauf für Abwher Spieler und für Angrif spieler wird der Vorfuß bevorzugt. Da es bei der vorliegenden Studie nur eine kurze Laufphase gab, kann auch diese dazu geführt haben, dass Probanden im Vorfußlauf liefen. Vorfuß Tischtennisspieler wechseln beim Spielen oftmals auch in den Rückfuß , aufgrund der Ermüdung der Plantarflexoren.( Hatala et al., 2013). 
Zur Beinasymmetrie beim Laufen existieren nur wenige biomechanische Analysen, die keine signifikanten Differenzen in der Kinematik und der Bodenreaktionskraft im Vergleich des dominanten vs. nicht-dominanten (bevorzugten) Beines vor bzw. nach Ermüdung (Brown et al.,2014; Hamil et al., 1984) ergaben. Ein Haupteffekt des Beines bei Rückfuß Tischtennisspieler bestand bei den Plantarflexoren beim maximalen isometrischen Drehmoment mit höheren Werten des linken Beins $(p=, 023)$ und damit auch beim Ermüdungsindex mit geringen Werten des linken Beines $(p=, 021)$.Das kann darauf zurückgeführt werden, dass das linke Bein das dominante Bein ist( Van der Harst et al.2007). Die stärkere Ermüdung der Dorsalflexoren des rechten Beines bei Rückfuß Tischtennisspieler kann aus einer insgesamt geringeren Kraftfähigkeit des nicht-dominanten Beines begründet werden. Bei den Plantarflexoren bestand zudem eine Wechselwirkung Bein*Fußaufsatz beim maximalen isometrischen Drehmoment ( $p=, 015$ ). Die Rückfuß Tischtennisspieler realisierten im Durchschnitt links höhere und rechts niedrigere Werte als die Vorfußläufer. Ein Grund dafür ist, dass die Rückfuß Tischtennisspieler das linke Bein als dominates Bein aufweisen im Gegensatz zu den Vorfußläufern, wo sich das rechte Bein als dominantes Bein zeigt. Dadurch zeigen sich Gegensätze in den Ergebnissen und können auf die unterschiedliche Beindominanz zurückgeführt werden(Van der Harst 2007).Der Links-/ Rechtsvergleich bei den Dorsalflexoren der Rückfuß Tischtennisspieler differierte beim maximalen isokinetischen Drehmoment im Ausdauertest $(\mathrm{p}=, 019)$ sowie beim Ermüdungsindex $(\mathrm{p}=, 010)$ mit höheren Werten des linken Beines. Dabei ist ebenfalls die Wechselwirkung Bein*Fußaufsatz $(\mathrm{p}=, 000)$ zu beachten. Die Asymmetrie in den Kraftvoraussetzungen der untersuchten Läufer mit höheren Werten des linken Beines(Rückfuß Tischtennisspieler)und mit höheren Werten des rechten Beines (Vorfuß Tischtennisspieler) sowohl der isometrischen Maximalkraft als auch der isokinetischen Kraftausdauer ist auf dessen Dominanz als Sprungbein zurückzuführen (Van der Harst et al., 2007).

Beide Gruppen absolvierten den Laufbandtest mit gleicher Laufgeschwindigkeit und vergleichbarer Doppelschrittlänge, Schrittlänge und Schrittfrequenz. Die Fußaufsatztechnik hat demnach keinen Einfluss auf die Doppelschrittlänge, Schrittlänge und Schrittfrequenz

Andere Studien zeigten, dass die Fußaufsatztechnik Einfluss auf die Schrittlänge und - frequenz hat. Liebermann et al.(2010) fanden aufgrund der stärkeren Kniebeugung der Vorfuß Tischtennisspieler eine verkürzte Schrittlänge, was auch in anderen Studien bestätigt wurde (Lieberman et al.2010; De Wit et al. 2000; Shih et al. 2013). In einer weiteren Untersuchung wurde eine verkürzte Schrittlänge gefunden und zusätzlich eine erhöhte Schrittfrequenz bei Vorfuß Tischtennisspieler (Derrick et al.,1998). Zusätzlich bedeutet eine höhere Schrittfrequenz, dass jeder Schritt weniger Zeit in Anspruch nimmt und daraus resultiert ein kürzerer Kontakt mit dem Boden, was auf die Bodenreaktionkräfte Einfluss hat( Squadrone et al. ,2009).

Diese Ergebnisse stimmen mit anderen Untersuchungen überein(Cavanagh et al.,1980; Ahn et al.,2014; Liebermann et al.,2012;Liebermann et al. 2010;Davis et al.,2010; Laughton et al.,2003; Squadrone et al.,2009; Hamill et al., 2011;Goss et al. 2012; Arendse et al.,2004; Shih et al., 2013 ; Williams et al. ,2000).Im Gegensatz zu Rückfuß Tischtennisspieler haben Vorfuß Tischtennisspieler ein geringeres Druckmaxima unter der Ferse und aufgrund der niedrigeren Belastung im Rückfußbereich auch ein geringeres Verletzungsrisiko( Daoud et al. 2012; Davis et al. 2010; Goss et al., 2012a; Liebermann et al., 2012;Liebermann et al. 2010).

Der Symmetrieindex der Druckverteilung unter der Ferse wies eine höhere Asymmetrie bei den Vorfuß Tischtennisspieler gegenüber Rückfuß Tischtennisspieler auf. Ein Grund dafür könnte sein, dass der Bodenkontakt bei Vorfuß Tischtennisspieler mit dem Fußballen stattfindet (Liebermann et al.,2010; Hasegawa et al. 2007; Fellin et al., 2010) welcher eine größere Fläche bietet als die Ferse. Beim Abrollen nutzen Vorfuß Tischtennisspieler daher nur einen geringen Teil der ohnehin kleineren Ferse. Die Rückfuß Tischtennisspieler nutzen beim ersten Bodenkontakt die Ferse komplett aus und rollen über die Fußballen ab. (Liebermann et al.,2010; Hasegawa et al. 2007; Fellin et al., 2010)

Die Ermüdung beeinflusst die Plantare Druckverteilung je nach Fußaufsatztechnik in unterschiedlicher Art und Weise (Weist et al., 2004;Nagel et al.,2008; Willson et al., 1999 ;Fan et al.,2011;Willems et al.,2012; García-Pérez et al. 2013).So konnten in einigen Studien eine erhöhte Vorfußbelastung nach Laufermüdung festgestellt werden (Bisiaux et al., 2008; Willems et al.,2012; 
Willson et al., 1999), welche auch nach lokaler Ermüdung der Plantar- und Dorsalflexoren gefunden wurden (Olivier et al., 2013). García-Pérez et al. (2013) berichten von einer erhöhten relativen Belastung des medialen Fußbogens und einem reduzierten Spitzendruck unter der seitlichen Ferse aufgrund der Ermüdung. Sie folgerten daraus, dass eine Veränderung des Abrollverhaltens als Folge von Ermüdung die plantare Druckverteilung verändert. Widersprüchliche Ergebnisse gab es zum Einfluss der Ermüdung durch Laufen bei der Druckverteilung der drei Fußregionen. Zum einen gab es einen signifikanten Anstieg des Spitzendrucks unter der Ferse(Weist, et al.,2004; Willems et al., 2012), zum anderen wurde auch ein Abfall des Spitzendrucks nach Ermüdung verzeichnet (Alfuth \& Rosenbaum, 2011; Bisiaux \& Moretto, 2008; Garcia-Perez et al., 2013; Willson \& Kernozek, 1999). Nach der lokalen Muskelermüdung waren die Werte des plantaren Druckmaximums unter der rechten Ferse $(p=0,02)$ niedriger, was daran liegen kann, dass das linke Bein stärker als das rechte Bein ist. Bei Vorfuß Tischtennisspieler gab es keine Veränderung, da der erste Bodenkontakt mit dem Fußballen erfolgt.Beide Läufergruppen zeigten nach Ermüdung niedrigere plantare Druckmaxima unter dem Vorfuß und bei den Rückfuß Tischtennisspieler zusätzlich unter der Ferse.Zur Erklärung der Reduktion des ersten Kraftmaximum und der maximalen Druckwerte unter der Ferse gibt es verschiedene Gründe. Zum einen führt eine reduzierte Vertikalbewegung des Körperschwerpunktes zu geringeren Landekräfte, zum anderen wird durch eine stärkere Beugung im Kniegelenk (Kellis et al., 2009) für eine bessere Schockabsorption gesorgt. Bei der Landung ist das Knie im Vorfußlauf stärker gebeugt und nicht gestreckt wie beim Rückfußlauf, womit eine bessere Schockabsorption der Aufprallkräfte zu einem geringeren Verletzungsrisiko bei Vorfuß Tischtennisspieler führt (Lieberman et al., 2010;De Wit et al., 2000;Shih et al., 2013).

Die Geschwindigkeit des Laufens beeinflusst die Fußaufsatztechnik des Läufers(Giandolini et al. ,2013;Nilsson et al. , 1989).Bezüglich der Laufgeschwindigkeit fanden Burnfield und Kollegen heraus, dass eine Erhöhung der Laufgeschwindigkeit zu erhöhten plantaren Spitzendruckwerten führt (Burnfield et al. 2004).

\section{Schlussfolgerung}

Das erste Kraftmaximum reduzierte sich bei beiden Tischtennis Gruppen nach dem Ermüdungsprotokoll im Vergleich zur Baseline im Durchschnitt in allen drei Laufgeschwindigkeiten. Bei den Rückfuß Tischtennisspieler traten höhere mittlere plantare Druckmaxima unter der Ferse bei zugleich geringen maximalen Druckwerten unter dem Mittel- und Vorfuß im Vergleich zu den Vorfuß Tischtennisspieler auf. Nach lokaler Ermüdung verringerte sich das plantare Druckmaximum bei den Rückfuß Tischtennisspieler unter der Ferse und unter dem Vorfuß Tischtennisspieler. Bei den Vorfuß Tischtennisspieler hat sich infolge der lokalen Ermüdung das plantare Druckmaximum unter der Ferse im Durschnitt erhöht und unter dem Vorfuß kam es zu einer Verringerung des plantaren Druckmaximums. Für weitere Untersuchungen ist es empfehlenswert längere Zeitabschnitte mit verschiedenen Geschwindigkeiten durchzuführen, um bessere Einblicke auf die Fußaufsatztechnik zu erhalten. Die optimale Lauftechnik ist dann vorhanden, wenn ein Läufer auf jede Situation mit einer entsprechenden Fußaufsatztechnik reagieren kann, das könnte zur Verringerung der Verletzungsgefahr beim Laufen führen. Für zukünftige Studien zur Ermüdung sollten Ermüdungsprotkolle für das Knie bzw. Inversion und Eversion des Sprunggelenks mit einbezogen werden. Bei weiteren Studien sollten auch die Mittelfußläufer mit untersucht werden um ein Vergleich der drei Fußaufsatztechniken auszuarbeiten. Die Probanden für weitere Studien sollten unterschiedlichen Geschlechts sein und die Altersgruppen sollten aus verschiedenen Altersklassen getestet werden.

\section{Literatur}

1.Ahn, A.N., Brayton, C., Bhatia, T., \& Martin, P. (2014). Muscle activity and kinematics of forefoot and rearfoot strike runners. Journal of Sport and Health Science, 3,102-112.

2.Alfuth, M., \& Rosenbaum, D. (2011). Long distance running and acute effects on plantar foot sensitivity and plantar foot loading. Neuroscience Letters, 503 (1), 58-62.

3.Altman, A.R., \& Davis I.S. (2012). A kinematic method for footstrike pattern detection in barefoot and shod runners. Gait \&Posture, 35,298-300. 
4.Arendse, R. E., Noakes, T. D., Azevedo, L. B., Romanov, N., Schwellnus, M. P., \& Fletcher, G. (2004). Reduced eccentric loading of the knee with the pose running method. Medicine and Science in Sports and Exercise, 36, 272-277.

5.Baker, J., Burnstein, A., \& Frankel, V. H. (1972). Fatigue fractures: Biomechanical considerations. Journal of Bone and Joint Surgery, 54, 1345.

6.Bennell, K., \& Brukner, P. (2005). Preventing and managing stress fractures in athletes. Physical Therapy in Sport, 6,171 - 180.

7.Bertelsen, M. L., Jensen, J. F., Nielsen, M. H., Nielsen, R. O., \& Rasmussen, S. (2013). Footstrike patterns among novice runners wearing a conventional, neutral running shoe. Gait \& posture, 38(2), 354-356.

8.Bisiaux, M., \& Moretto, P. (2008). The effects of fatigue on plantar pressure distribution in walking. Gait \& posture, 28(4), 693-698.

9.Bobbert, M.F., Yeadon, M.R., \& Nigg, B.M. (1992). Mechanical analysis of the landing phase in heel-toe running. Journal of Biomechanics ,25,223-34.

10.Bramble, D.M., Lieberman, D.E. (2004). Endurance running and the evolution of Homo. Nature, 432,345-52.

11.Bredeweg, S., \& Buist, I. (2011). No relationship between running related injuries and kinetic variables. British Journal of Sports Medicine, 45 (4), 328-328.

12.Brown, A. M., Zifchock, R. A., \& Hillstrom, H. J. (2014). The effects of limb dominance and fatigue on running biomechanics. Gait \& posture,39(3), 915-919.

13.Burnfield, J.M., Few, C.D., Mohamed, O.S., \& Perry, J. (2004). The influence of walking speed and footwear on plantar pressures in older adults. Clinical Biomechanics ,19, 78-84.

14.Burr, D. B. (1997). Bone exercise and stress fractures. Exercise and Sport Sciences Reviews, 25, 171-194.

15.Cavanagh, P.R. \& Lafortune, M.A. (1980). Ground reaction forces in distance running. Journal of Biomechanics, 13(5),397-406.

16.Christina, K. A., White, S. C., \& Gilchrist, L. A. (2001). Effect of localized muscle fatigue on vertical ground reaction forces and ankle joint motion during running. Human movement science, 20(3), 257-276.

17.Cohen, J. (1988). Statistical power analysis for behavior sciences.

18.Cohen, J. (1992). Statistic a power primer. Psychology Bulletin, 155-159.

19.Daoud, A. I., Geissler, G. J., Wang, F., Saretsky, J., Daoud, Y. A., \& Lieberman, D. E.(2012). Foot strike and injury rates in endurance runners: a retrospective study. Medicine and Science in Sports and Exercise, 44, 1325-1334.,

20.Davis, I. S., Bowser, B., \& Mullineaux, D. (2010). Do impacts cause running injuries? Aprospective investigation.Conference Proceedings of the Annual Meeting of the American,763

21.De Almeida, M.O., Saragiotto B.T., Yamato T.P. , \& Lopes A.D.(2015). Is the rearfoot pattern the most frequently foot strike pattern among recreational shod distance runners. Physical Therapy in Sport, 16(1), 29-33.

22.Derrick, T.R., Hamill, J., \& Caldwell, G.E. (1998). Energy absorption of impacts during running at various stride lengths. Medicine and Science in Sports and Exercise, 30,128-35.

23.De Wit, B., De Clercq, D., \& Aerts P.(2000). Biomechanical analysis of the stance phase during barefoot and shod running, Journal of Biomechanics,33,269-78.

24.Dickhuth, H.H., Mayer, F., Grau, S., Baur, H., Hirschmüller, A., Horstmann, T. \& Gollhofer, A.(2001). Verletzungen und Beschwerden im Laufsport. Deutsches Ärzteblatt, 98(19),12541259.

25.Dickinson, J. A., Cook, S. D., \& Leinhardt, T. M. (1985). The measurement of shock waves following heel strike while running. Journal of Biomechanics, 18, 415-422.

26.Divert, C., Mornieux, G., Baur ,H., Mayer, F., \& Belli , A.(2005). Mechanical comparison of barefoot and shod running. International Journal of Sports Medicine,26,593-8.

27.Divert C., Mornieux, G., Freychat, P., Baly, L., Mayer,F., \& Belli, A. (2008). Barefootshod running differences: shoe or mass effect?. International Journal of Sports Medicine , 229,512-8.

28.Fan, Y., Li, Z., Lv, C., \& Luo, D. (2011) Natural Gaits of the Non-Pathological Flat Foot and High-Arched Foot. PLOS ONE ,6(3). 
29.Fellin, R. E., Rose,W. C., Royer, T. D., \& Davis, I. S. (2010). Comparison of methods for kinematic identification of footstrike and toe-off during overground and treadmill running. Journal of Science and Medicine in Sport, 13, 646-650.

30.Flynn, J. M., Holmes, J.D., Andrews, D.M. (2004). The effect of localized leg muscle fatigue on tibial impact acceleration. Clinical,19(7),726-732.

31.Fredericson, M., \& Misra, A. K. (2007). Epidemiology and aetiology of marathon running injuries. Sports Medicine, 37(4-5), 437-439.

32.García-Pérez, J. A., Pérez-Soriano, P., Llana, S., Martínez-Nova, A., \& Sánchez-Zuriaga, D. (2013). Effect of overground vs treadmill running on plantar pressure: Influence of fatigue. Gait \& posture, 38(4), 929-933.

33.Gerlach, K. E., White, S. C., Burton, H. W., Dorn, J. M., Leddy, J. J. and Horvath, P. J. (2005). Kinetic Changes with Fatigue and Relationship to Injury in Female Runners. Medicine and Science in Sports and Exercise, 37(4),657-663.

34.Giandolini, M., Arnal, P. J., Millet, G. Y., Peyrot, N., Samozino, P., Dubois, B. (2013). Impact reduction during running: efficiency of simple acute interventions in recreational runners. European Journal of Applied Physiology, 113, 599-609.

35.Girard, O., Millet, G., Slawinski, J., Racinais, S., \& Micallef, J. P. (2010). Changes in leg-spring behavior during a 5000m self-paced run in differently trained athletes. Science \& Sports, 25(2), 99-102.

36.Goss, D. L., \& Gross, M. T. (2012). A review of mechanics and injury trends among various running styles. US Army Medical Department Journal, 62-71

37.Goss, D. L., \& Gross, M. T. (2012a). Relationships among self-reported shoe type, footstrike pattern, and injury incidence. US Army Medical Department Journal, 25-30

38.Grimston, S. K., \& Zernicke, R. F. (1993). Exercise-Related Stress Responses in Bone. Journal of Applied Biomechanics, 9(1).

39.Hamill, J., Bates, B. T., \& Knutzen, K. M. (1984). Ground reaction force symmetry during walking and running. Research Quarterly for Exercise and Sport, 55(3), 289-293.

40.Hamill, J., Russell, E.M., Gruber, A.H., \& Miller, R.(2011). Impact characteristics in shod and barefoot running. Footwear Science,3,33-40.

41.Hasegawa, H., Yamauchi, T. \& Kraemer,W.J.(2007). Foot strike patterns of runners at the $15-\mathrm{km}$ point during an elite-level half marathon. Journal of Strength \& Conditioning Research, 21(3),888-893.

42.Hatala, K.G., Dingwall, H.L., Wunderlich, R.E., \& Richmond, B.G. (2013). Variation in Foot Strike Patterns during Running among habitually barefoot populations.PLoS ONE, 8 (1),525-528.

43.Hong, Y., Wang, L., Li, J. X., \& Zhou, J. H. (2012). Comparison of plantar loads during treadmill and overground running. Journal of Science and Medicine in Sport, 15(6), 554-560.

44.Hreljac, A. (2005). Etiology, prevention, and early intervention of overuse injuries in runners: a biomechanical perspective. Physical medicine and rehabilitation clinics of North America, 16(3), 651-667.

45.James, S.L.,Bates, B.T., \& Osternig, L.R.(1978). Injuries to runners. American Journal of Sports Medicine, 2,97-98.

46.Kellis, E. \& Kouvelioti, V. (2009). Agonist versus antagonist muscle fatigue effects on thigh muscle activity and vertical ground reaction during drop landing. Journal of Electromyography and Kinesiology, 19(1),55-64.

47.Kellis, E., Zafeiridis, A., \& Amiridis, I.G. (2011). Muscle coactivation before and after the impact phase of running following isokinetic fatigue. Journal of Athletic Training ,46(1),11-9.

48.Kerr, B., Beauchamp, L., Fikhkr, V., \& Neil, R. (1983). Foot strike patterns in distance running. Biomechanical aspects of sport shoes and playing surface, International Symposium on Biomechanical Aspects of Sports and Playing Surfaces.

49. Kleindienst, F. I. , Campe, S. , Graf, E. S. , Michel, K.J. , \& Witte, K. (2007).Differences between fore- and rearfoot strike running patterns based on kinetics and kinematics. XXV ISBS Symposium Ouro Preto - Brazil.

50.Kulmala, J., Pasanen, K. , Parkkari, J.(2013) Forefoot strikers exhibit lower running-induced knee loading than rearfoot strikers. Medicine and Science in Sports and Exercise, 45(12):2306-2313. 
51.Landreneau , L., Watts, K., Heitzman, J.E. , \& Lee W. (2014)Lower limb muscle activitz during forefoot and rearfoot strike running techniques. International Journal Sports Physical Therapy,9(7),888-897.

52.Laughton,. CA., Davis, I.M., \& Hamill, J.(2003). Effect of strike pattern and orthotic intervention on tibial shock during running. Journal of Applied Biomechanics ,19,153-68.

53.Lieberman, D. E., Venkadesan, M., Werbel, W. A., Daoud, A. I., D’Andrea, S.,\& Davis, I. S.(2010). Foot strike patterns and collision forces in habitually barefoot versus shod runners. Nature, 463, 531-535.

54.Lieberman ,D.E. (2012). What we can learn about running from barefoot running: an evolutionary medical perspective. Exercise and Sport Sciences Reviews, 40,63-72.65

55.Liebl, D., Willwacher, S., Hamill, J., \& Brügemann G.P.(2014). Ankle plantarflexion strength in rearfoot and forefoot runners: a novel clusteranalytic approach, Human Movement Science,35,104-120.

56. Marquardt, M. (2002). Natürlich Laufen. Spomedis, 14-36.

57. Mattes, K., Hazzaa, W.E. A., Schaffert, N., \& Manzer, S.(2015) Local concentric muscle fatigue of the ankle dorsiflexors and plantar flexors: A reproducibility study. Isokinetics and Exercise Science, 23,87-92.

58.Milner ,C.E., Ferber, R., Pollard, C.D., Hamill, J., Davis, I.S.(2006) Biomechanical factors associated with tibial stress fracture in female runners. Medicine \& Science in Sports\& Exercise,38(2),323-328.

59.Mizrahi, J., Verbitsky, O., Isakov, E., \& Daily, D. (2000). Effect of fatigue on leg kinematics and impact acceleration in long distance running. Human movement science, 19(2), 139-151.

60.Nagel, A., Fernholz, F., Kibele, C., \& Rosenbaum, D. (2008). Long distance running increases plantar pressures beneath the metatarsal heads: a barefoot walking investigation of 200 marathon runners. Gait \& posture, 27(1), 152-155.

61.Nigg, B. M., Cole, G. K., \& Brüggemann. C. P. (1995). Impact forces during heel-toe running. Journal of Applied Biomechanics, 11, 07432.

62.Nilsson, J., \& Thorstensson, A. (1989). Ground reaction forces at different speeds of human walking and running. Acta Physiologica Scandinavica, 136, 217-227.

63.Nummela, A., Rusko, H., \& Mero, A. (1994). EMG activities and ground reaction forces during fatigued and nonfatigued sprinting. Medicine \& Science in Sports \& Exercise,26(5), 605-609.

64.Novacheck ,T.F.,(1998). The biomechanics of running. Gait Posture, 7,77-95.

65.Nyland, J. A., Shapiro, R., Stine, R. L., Horn, T. S., \& Ireland. M. L. (1994). Relationship of fatigued run and rapid stop to ground reaction forces, lower extremity kinematics, and muscle activation. Journal of Orthopaedic and Sports Physical Therapy, 20, 132-137.

66.Olivier, P. (2013). Effect of invertor/evertor and plantar-/dorsiflexor fatigue on plantar pressure distribution. South African Journal for Research in Sport, Physical Education and Recreation, 35(1), 143-152.

67.Perl, D.P., Daoud ,A.I.,\& Lieberman, D.E.(2012).Effects of footwear and strike type on running economy. Medicine \& Science in Sports \& Exercise,44,1335-4366.

68.Pohl, M.B., Hamill, J.,\& Davis, I.S.(2009). Biomechanical and anatomic factors associated with a history of plantar fasciitis in female runners. Clinical Journal of Sport Medicine,19(5),372-376.

69.Rabita, G., Slawinski, J., Girard, O., Bignet, F., \& Hausswirth, C. (2011). Spring-mass behavior during exhaustive run at constant velocity in elite triathletes. Medicine and science in sports and exercise, 43(4), 685-692.

70.Reber, L., Perry, J., \& Pink, M. (1993). Muscular control of the ankle in running. The American Journal of Sports Medicine, 21(6), 805-810.

71.Richards, C.E.,Magin , P.J., \& Callister, R. (2009). Is your prescription of distance running shoes evidence-based?.British Journal of Sports Medicine,43(3),159-62.

72.Riley, P. O., Dicharry, J., Franz, J. A. S. O. N., Croce, U. D., Wilder, R. P., \& Kerrigan, D. C. (2008). A kinematics and kinetic comparison of overground and treadmill running. Medicine and Science in Sports and Exercise, 40(6), 1093.

73.Roberts, T.J.,\& Azizi, E.(2011) Flexible mechanisms: the diverse roles of biological springs in vertebrate movement. Journal of Experimental Biology,214,353-361. 
74.Rooney, B.D., \& Derrick, T.R.(2013). Joint contact loading in forefoot and rearfoot strike patterns during running. Journal of Biomechanics,46,2201-2206.

75.Schache, A. G., Blanch, P. D., Rath, D. A., Wrigley, T. V., Starr, R., \& Bennell, K. L. (2001). A comparison of overground and treadmill running for measuring the three-dimensional kinematics of the lumbo-pelvic-hip complex. Clinical Biomechanics, 16(8), 667-680.

76.Shih, Y., Lin, K. L., \& Shiang, T. Y. (2013). Is the foot striking pattern more important than barefoot or shod conditions in running?. Gait and Posture, 38, 490-494

77.Slawinski, J., Heubert, R., \& Quievre, J. (2008). Changes in spring-mass model parameters and energy cost during track running to exhaustion.Journal of Strength \& Conditioning Research, 22(3),930-936.

78.Squadrone,R., \& Gallozzi C.(2009). Biomechanical and physiological comparison of barefoot and two shod conditions in experienced barefoot runners. Journal of Sports Medicine and Physical Fitness,49,6-13.

79.Van der Harst, J. J., Gokeler, A., \& Hof, A. L. (2007). Leg kinematics and kinetics in landing from a single-leg hop for distance. A comparison between dominant and non-dominant leg. Clinical Biomechanics, 22(6), 674-680.

80.van Mechelen, W. (1992). Running injuries. Sports Medicine, 14(5), 320-335.

81.Walther, M. (2005). Vorfußlaufen schützt nicht vor Überlastungsproblemen. Orthopädieschuhtechnik, 6, 34 .

82.Weist, R., Eils, E., \& Rosenbaum, D.(2004). The influence of muscle fatigue on electromyogram and plantar pressure patterns as an explanation for the incidence of metatarsal stress fractures.American Journal of Sports Medicine,32(8),1893-1898.

83.Williams, D. S., McClay, I. S., \& Manal, K. T. (2000). Lower extremity mechanics in runners with a converted forefoot strike pattern. Journal of Applied Biomechanics, 16, 210-218

84.Willems, T. M., De Ridder, R., \& Roosen, P. (2012). The effect of a long-distance run on plantar pressure distribution during running. Gait \& posture, 35(3), 405-409.

85.Willson, J. D., \& Kernozek, T. W. (1999). Plantar loading and cadence alterations with fatigue. Medicine and science in sports and exercise, 31(12), 1828-1833.

86.Zadpoor, A. A., \& Nikooyan, A. A. (2011). The relationship between lower-extremitystress fractures and the ground reaction force: a systematic review. Clinical Biomechanics, 26, 23-28.

87.Zifchock, R. A., Davis, I., Higginson, J., \& Royer, T. (2008). The symmetry angle: a novel, robust method of quantifying asymmetry. Gait \& posture, 27(4), 622-627. 